\title{
NÍVEL DE ATIVIDADE FÍSICA E FATORES ASSOCIADOS EM COLABORADORES
}

\author{
João Marcos de Paula Lopes ${ }^{1 *}$, Mônica Thais Soares Macedo ${ }^{1}$, Ataualba Ramalho de \\ Meirelles Filho' \& Josiane Santos Brant Rocha ${ }^{1}$
}

\begin{abstract}
RESUMO
LOPES, J.M.P.; MACEDO, M.T.S.; MEIRELLES FILHO, A.R.; ROCHA, J.S.B. Nível de atividade física e fatores associados em colaboradores. Perspectivas Online: Biológicas \& Saúde, v.11, n.37, p.50-61, 2021.
\end{abstract}

A atividade física é todo o movimento corporal sem intencionalidade que propicie o gasto energético acima do nível de repouso, visto que esse conceito a diferencia do exercício físico que é planejado, estruturado e repetitivo. Diante disso, o objetivo desse estudo foi estimar o nível de atividade física e os fatores associados em colaboradores de um Centro Universitário. Trata-se de um estudo transversal com 128 colaboradores técnicos, selecionados por amostragem probabilística, entre janeiro a dezembro de 2019. Além da variável desfecho, atividade física, foram avaliados os fatores sociodemográficos, hábitos comportamentais e perfil antropométrico. Foram realizadas análises descritivas das variáveis investigadas através de suas distribuições de frequências. Posteriormente, foram realizadas análises bivariadas através da regressão de Poisson, e para as análises múltiplas utilizou-se regressão de Poisson hierarquizado para identificar fatores associados a atividade física. A prevalência de prática de atividade física foi de $14,8 \%$ dos colaboradores. Os fatores idade $(\mathrm{p}=0,041)$, qualidade do sono $(\mathrm{p}=0,139)$, estilo de vida $(\mathrm{p}=0,026)$, problema na coluna $(\mathrm{p}=0,116)$ e IMC $(\mathrm{p}=, 041) \quad$ apresentaram-se como predisponentes para a p'rática insuficiente de atividade física. No entanto, o fator ser casado apresentou-se como efeito protetor para a atividade física. Concluindo, o baixo nível de atividade física está relacionado a fatores como sexo, sono e estilo de vida. Além disso, nesse estudo pode-se constatar que os colaboradores casados apresentam efeito protetor para a prática de atividade física.

Palavras-chave: Exercício Físico; Qualidade de Vida; Trabalhadores; Adulto.

\footnotetext{
${ }^{1}$ Universidade Estadual de Montes Claros - UNIMONTES - Av. Prof. Rui Braga, s/n, Vila Mauricéia, Montes Claros, MG, CEP: 39401-089, Brasil. (*) e-mail: joaomarcosdepaula@ yahoo.com.br

Data de recebimento: 29/01/2021. Aceito para publicação: 21/06/2021 .

Data publicação: 30/06/2021.
} 


\title{
LEVEL OF PHYSICAL ACTIVITY AND ASSOCIATED FACTORS IN EMPLOYEES \\ João Marcos de Paula Lopes ${ }^{1 *}$, Mônica Thais Soares Macedo' ${ }^{1}$, Ataualba Ramalho de Meirelles Filho ${ }^{1}$ \& Josiane Santos Brant Rocha ${ }^{1}$
}

\begin{abstract}
LOPES, J.M.P.; MACEDO, M.T.S.; MEIRELLES FILHO, A.R.; ROCHA, J.S.B. Nível de atividade física e fatores associados em colaboradores. Perspectivas Online: Biológicas \& Saúde, v.11, n.37, p.50-61, 2021.
\end{abstract}

Physical activity is any unintentional body movement that promotes energy expenditure above the resting level, since this concept differentiates it from the physical exercise that is planned, structured and repetitive. Therefore, the objective of this study was to estimate the level of physical activity and associated factors in employees of a University Center. This is a cross-sectional study with 128 technical collaborators, selected by probabilistic sampling, between January and December 2019. In addition to the outcome variable, physical activity, sociodemographic factors, behavioral habits and anthropometric profile were evaluated. Descriptive analyzes of the investigated variables were carried out through their frequency distributions. Subsequently, bivariate analyzes were performed using Poisson regression, and for multiple analyzes, hierarchical Poisson regression was used to identify factors associated with physical activity. The prevalence of physical activity was $14.8 \%$ of employees. The factors age $(\mathrm{p}=0.041)$, quality of sleep $(\mathrm{p}=0.139)$, lifestyle $(0.026)$, spinal problems $(0.116)$ and BMI $(\mathrm{p}=0.041)$ were found to be predisposing to insufficient physical activity. However, being married was a protective effect for physical activity. In conclusion, the low level of physical activity is related to factors such as sex, sleep and lifestyle. In addition, in this study it can be seen that married employees have a protective effect for the practice of physical activity.

Keywords: Physical Exercise; Quality of Life; Workers; Adult.

${ }^{1}$ Montes Claros State University - UNIMONTES - Av. Rui Braga, s/n, Vila Mauricéia, Montes Claros, MG, Postal Code: 39401-089, Brazil. (*) e-mail: joaomarcosdepaula@yahoo.com.br

Received: 01/29/2021. Accepted: 06/21/2021.

Publication in: 30/06/2021.

Persp. Online: biol. \& saúde, Campos dos Goytacazes, 37 (11) 50-61, 2021

seer.perspectivasonline.com.br 


\section{INTRODUÇÃO}

A atividade física é todo o movimento corporal sem intencionalidade que propicie o gasto energético acima do nível de repouso, visto que esse conceito a diferencia do exercício físico que é planejado, estruturado e repetitivo (ARAÚJO; OLIVEIRA, 2019). Entre adultos, os benefícios da AF podem ser ainda mais evidentes, pois reduz a ocorrência de doenças crônicas não transmissíveis (DNCT), quedas e limitações funcionais e, consequentemente, leva ao aumento da expectativa de vida e melhoria da qualidade de vida desse grupo (PEIXOTO et al., 2018; BATISTA; SILVA; CALOMENI, 2018). De acordo com Talarico Neto et al. (2020), em níveis insuficientes, a falta da prática de atividade física pode estar relacionada ao acomentimento de doenças como a diabetes, obesidade e até mesmo o câncer dentre outras.

Evidências demonstram que o incentivo a prática de exercício físico pode diminuir os gastos investidos em assistência médica, diminuição das faltas no trabalho, na demissão de funcionários e até mesmo no aumento da produtividade, pois a prática suficiente e regular de atividade física implica na prevenção diversas patologias (CORRÊA et al., 2019).

No entanto, apesar do conhecimento sobre os benefícios e efeitos positivos da atividade física, sua prática ainda é pouco valorizada (SANCHEZ et al., 2014). De acordo com a Organização Mundial da Saúde (OMS) (2018), em 2018, em todo o mundo, 20\% da população não praticava exercícios com frequência e intensidade adequadas para a sua faixa etária, além dessa insuficiência ativa ser responsável por $71 \%$ de todas as mortes no mundo.

Esses fatos demonstram que conhecer os diversos fatores associados ao baixo nível de atividade física torna-se essencial, a fim de rastrear os possíveis acometimentos de doenças crônicas não-transmissíveis, além de propor políticas públicas de intervenção e promoção da saúde visando a qualidade de vida dessa população.

Diante disso, esse estudo tem como objetivo estimar o nível de atividade física e os fatores associados em colaboradores técnicos de um Centro Universitário.

Persp. Online: biol. \& saúde, Campos dos Goytacazes, 37 (11) 50-61, 2021

seer.perspectivasonline.com.br 


\section{METODOLOGIA}

Trata-se de um estudo epidemiológico, transversal e analítico, originado de um projeto intitulado "Condições de saúde dos colaboradores técnicos de uma instituição de ensino superior", realizado no Centro Universitário Pitágoras (UNIFIPMoc), em Montes Claros - MG, no período de janeiro a dezembro de 2019.

A população do estudo foi composta por 138 colaboradores do Centro Universitário UNIFIPMoc, distribuídos no Núcleo de Atenção de Práticas Profissionalizantes (NASPP), no Centro de Prática de Engenharia, Arquitetura e Gestão (CEPEAGE) e no Núcleo de Práticas Jurídicas (NPJ). O tamanho amostral foi determinado em função dos múltiplos agravos à saúde dos colaboradores investigados na pesquisa. Considerou-se uma prevalência máxima esperada de $50 \%$, com nível de confiança de $95 \%$ e margem de erro de $5 \%$. No entanto, pensando em possíveis perca de dados durante o processo de coleta, foram recrutados 128 indivíduos que compuseram a amostra final dessa pesquisa.

A seleção da amostra foi do tipo probabilístico em um único estágio. Foram selecionados para participação no estudo todos os colaboradores que atuavam no Campus sede, e nos três centros que integram a instituição. Dessa forma, foram incluídos todos os colaboradores em exercício da função há pelo menos um ano, sendo excluídos os colaboradores em desvio de função ou em licença médica por qualquer natureza.

Inicialmente foram realizadas reuniões pré-agendadas com o diretor da UNIFIPMoc, a fim de sensibilizar os colaboradores quanto à importância do estudo e da sua participação e orientar sobre os procedimentos da pesquisa. A coleta de dados ocorreu no período de fevereiro a abril de 2019, nas dependências da UNIFIPMoc, NASPP, CEPEAGE, e NPJ por uma equipe previamente capacitada.

Foi investigada a variável dependente (prática de atividade física) por meio do International Physical Activity Questionnaire (IPAC), no qual inicialmente categoriza em sedentária, ativa e muito ativa. Posteriormente essa variável foi dicotomizada em sedentária e a categoria ativa e muito ativa foi agrupada em ativa.

A versão curta desse questionário consta com oito perguntas relacionadas às atividades físicas realizadas na última semana por, pelo menos, 10 minutos contínuos anterior à aplicação do questionário. Segundo a classificação do instrumento, considera-se sedentária a que não realizou nenhuma atividade física por pelo menos 10 minutos contínuos durante a semana. Insuficientemente ativa são as que realizam atividade física por pelo menos 10 minutos por semana, porém, insuficiente para ser classificado como ativo. Pode ser divido em dois grupos: faz atividade cinco dias por semana ou durante 150 minutos por semana. Para ser considerado ativo, deve se cumprir recomendações: mais que três dias por semana e mais que 20 minutos por sessão de atividade vigorosa ou mais de cinco dias por semana e mais que 30 minutos por sessão de atividade moderada ou mais de cinco dias por semana e mais que 150 minutos por semana de caminhada, atividade moderada ou vigorosa. A pessoa muito ativa é a que fez atividade vigorosa por mais de que cinco dias por semana e 30 minutos por sessão ou mais que três dias por semana e 20 minutos por sessão de atividade moderada ou caminhada.

Persp. Online: biol. \& saúde, Campos dos Goytacazes, 37 (11) 50-61, 2021

seer.perspectivasonline.com.br 
Os colaboradores responderam questões referentes às variáveis independentes (sociodemográficos, hábitos comportamentais e perfil antropométrico). As variáveis sociodemográficas incluíram: Idade (até 35 anos e maior de 35 anos), sexo (masculino e feminino), cor da pele (branca e não branca), curso mais elevado (superior e fundamental/médio), estado conjugal (com companheiro e sem companheiro), outra atividade (não e sim), interesse em sair do ramo (sem interesse e com interesse).

As variáveis que compreenderam os Hábitos Comportamentais: estado de saúde (bom e regular), diabetes (sem diabetes e com diabetes), cigarros (não fuma e fuma), pressão alta (não e sim), álcool (não bebo e bebo), sono alterado (não e sim) estilo de vida fantástico (excelente e regular), colesterol (não e sim), problema na coluna (não e sim).

A variável relacionada ao Perfil Antropométrico foi: Índice de Massa Corpórea (IMC) (eutrófico e sobrepeso/obeso).

O estilo de vida foi avaliado através do questionário validado "Estilo de Vida Fantástico" o qual consiste em um instrumento genérico desenvolvido no Departamento de Medicina Familiar da Universidade McMaster, no Canadá, por Wilson e Ciliska em 1984, com a finalidade de possibilitar uma forma de mensurar o estilo de vida dos indivíduos. A origem da palavra "fantástico" vem do acrônimo FANTASTIC representando as iniciais dos nove domínios distribuídos em 25 questões os quais são: Family and friends (família e amigos); A = Activity (atividade física); $\mathrm{N}=$ Nutrition (nutrição); $\mathrm{T}=$ Tobacco \& toxics (cigarro e drogas); A = Alcohol (álcool); $S$ = Sleep, seatbelts, stress, safe sex (sono, cinto de segurança, estresse e sexo seguro); $\mathrm{T}$ = Type of behavior (tipo de comportamento; padrão de comportamento $\mathrm{A}$ ou B); I = Insight (introspecção); C = Career (trabalho; satisfação com a profissão). As somas de todos os pontos do questionário são classificadas da seguinte forma: excelente (85-100 pontos); muito bom (70-84 pontos); bom (55-69 pontos); regular (35-54 pontos); necessita melhorar (034 pontos). Posteriormente foi dicotomizado em bom (35-100 pontos) regular (0-34 pontos) (ANEZ; REIS; PETROSKI, 2008).

O cálculo do índice de massa corporal (IMC) foi realizado utilizando a mensuração da estatura com auxílio do estadiômetro da marca SECA 206, com escala de 0 a 2,20 m, previamente fixado numa parede plana com noventa graus em relação ao chão e sem rodapés. O peso foi aferido utilizando a Balança de Controle Corporal OMRON HBF-514C Digital. Esse índice é calculado pela massa do indivíduo dividida pelo quadrado de sua estatura, em que a massa é em quilogramas e a estatura em metros. Os pontos de corte foram considerados segundo normas específicas, sendo o IMC considerado normal numa faixa de $18,5-24,9 \mathrm{Kg} / \mathrm{m}^{2}$, determinando um indivíduo eutrófico; o sobrepeso é identificado numa faixa de IMC 25-29,9 $\mathrm{Kg} / \mathrm{m}^{2}$; já a obesidade é caracterizada por IMC acima de $30 \mathrm{Kg} / \mathrm{m}^{2}$ (ASSOCIAÇÃO BRASILEIRA PARA ESTUDO DA OBESIDADE E DA SÍNDROME METABÓLICA, 2016). Posteriormente foi dicotomizado em eutrófico $\left(\leq 24,9 \mathrm{Kg} / \mathrm{m}^{2}\right)$ e Sobrepeso/Obeso $(>24,9$ $\left.\mathrm{Kg} / \mathrm{m}^{2}\right)$.

Inicialmente, foram realizadas análises descritivas das variáveis investigadas por meio de suas distribuições de frequências. Em seguida, foram realizadas análises bivariadas por meio do teste qui-quadrado e as variáveis associadas à prática de atividade física até o nível de significância de $25 \%$ ( $\mathrm{p} \leq 0,25)$, permaneceram no modelo para analise multivariada. No modelo ajustado final, estimado através da Regressão Logística Binária, foram mantidas as variáveis que apresentaram um nível de significância de até $5 \%(\mathrm{p} \leq 0,05)$.

Persp. Online: biol. \& saúde, Campos dos Goytacazes, 37 (11) 50-61, 2021

seer.perspectivasonline.com.br 
A pesquisa atendeu aos princípios éticos da Resolução do Conselho Nacional de Saúde (CNS) n466/2012 sendo aprovado pelo Comitê de Ética em Pesquisa parecer $n^{\circ} 3.060 .579$ (CEP/UNIFIPMoc, 39408-007). Todos os participantes da pesquisa receberam, concordaram e assinaram o Termo de Consentimento Livre e Esclarecido.

\section{RESULTADOS}

Após as perdas, a amostra foi composta por 128 colaboradores técnicos, com uma média de idade de 35,5 \pm 9,95, dos quais 14,8\% dos colaboradores relataram apresentar prática suficiente de atividade física, como explicitado no gráfico 1.

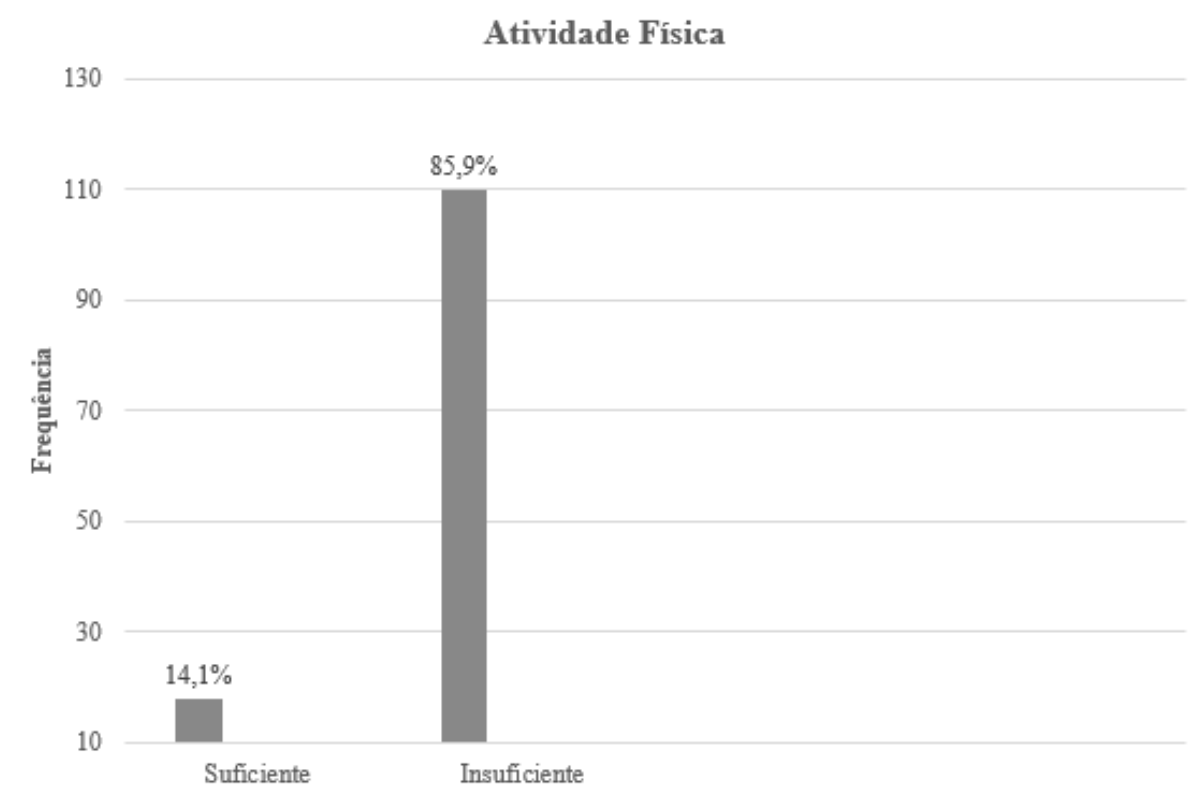

Gráfico 1: Nível de atividade física.

As associações significativas ao nível $\mathrm{p}<0,25$ para a prática de atividade física estão dispostos na (tabela 1). Ao analisar o perfil sociodemográfico os hábitos comportamentais e o perfil antropométrico dos colaboradores técnicos com a prática suficiente de atividade física, observou-se associação, na análise bivariada, considerando os aspectos sociodemográficos apenas para idade $(\mathrm{p}=0,041)$, estado conjugal $(\mathrm{p}=0,006)$. Analisando os hábitos comportamentais dos colaboradores técnicos, observou-se que as variáveis que apresentaram associadas foram: Baixa qualidade do sono $(\mathrm{p}=0,139)$ estilo de vida $(\mathrm{p}=0,026)$ e problema de coluna $(\mathrm{p}=0,116)$. Observando os dados obtidos no perfil antropométrico, observou-se que apenas IMC associou-se ( $\mathrm{p}=0,041)$ com a pratica suficiente de educação física (Tabela 1).

Os fatores sociodemográficos (idade, cor, curso mais elevado, realiza outra atividade, tem interesse em sair do ramo), hábitos comportamentais (estado de saúde, diabetes, pressão alta, uso de cigarros, álcool, colesterol, depressão) e perfil antropométrico (IMC) não apresentaram associações significativas $\mathrm{p}<0,250 \mathrm{com}$ a presença da prática suficiente de atividade física, não sendo incluídos no modelo.

Persp. Online: biol. \& saúde, Campos dos Goytacazes, 37 (11) 50-61, 2021

seer.perspectivasonline.com.br 
Tabela 1: Caracterização da amostra e razão de prevalência (RP) bruta para atividade física de acordo com os fatores sociodemográficos, hábitos comportamentais e perfil antropométrico.

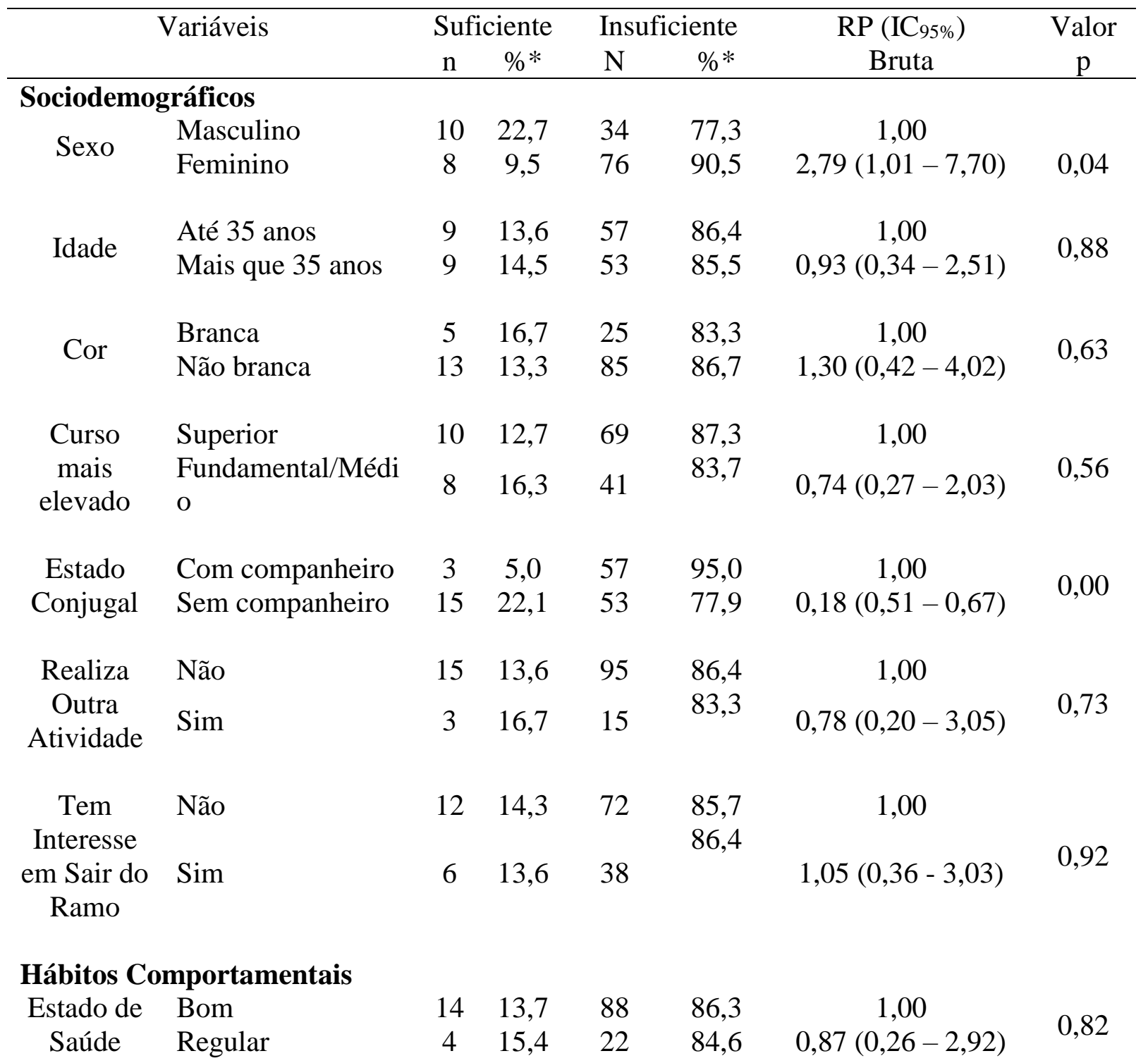

Persp. Online: biol. \& saúde, Campos dos Goytacazes, 37 (11) 50-61, 2021

seer.perspectivasonline.com.br 


\begin{tabular}{|c|c|c|c|c|c|c|c|}
\hline Diabetes & $\begin{array}{l}\text { Não } \\
\text { Sim }\end{array}$ & $\begin{array}{c}1 \\
17\end{array}$ & $\begin{array}{l}25,0 \\
13,7\end{array}$ & $\begin{array}{c}3 \\
107\end{array}$ & $\begin{array}{l}75,0 \\
86,3\end{array}$ & $\begin{array}{c}1,00 \\
2,09(0,20-21,35)\end{array}$ & 0,52 \\
\hline Cigarros & $\begin{array}{l}\text { Não Fumo } \\
\text { Fumo }\end{array}$ & $\begin{array}{c}14 \\
4\end{array}$ & $\begin{array}{l}13,0 \\
20,0\end{array}$ & $\begin{array}{l}94 \\
16\end{array}$ & $\begin{array}{l}87,0 \\
80,0\end{array}$ & $\begin{array}{c}1,00 \\
0,59(0,17-2,04)\end{array}$ & 0,40 \\
\hline $\begin{array}{l}\text { Pressão } \\
\text { Alta }\end{array}$ & $\begin{array}{l}\text { Não } \\
\text { Sim }\end{array}$ & $\begin{array}{c}14 \\
4\end{array}$ & $\begin{array}{l}13,0 \\
20,0\end{array}$ & $\begin{array}{l}94 \\
16\end{array}$ & $\begin{array}{l}87,0 \\
80,0\end{array}$ & $\begin{array}{c}1,00 \\
0,59(0,17-2,04)\end{array}$ & 0,40 \\
\hline Álcool & $\begin{array}{l}\text { Não Bebo } \\
\text { Bebo }\end{array}$ & $\begin{array}{c}8 \\
10\end{array}$ & $\begin{array}{l}12,5 \\
15,6\end{array}$ & $\begin{array}{l}56 \\
54\end{array}$ & $\begin{array}{l}87,5 \\
84,4\end{array}$ & $\begin{array}{c}1,00 \\
0,77(0,28-2,10)\end{array}$ & 0,61 \\
\hline $\begin{array}{l}\text { Sono } \\
\text { alterado }\end{array}$ & $\begin{array}{l}\text { Não } \\
\text { Sim }\end{array}$ & $\begin{array}{c}12 \\
6\end{array}$ & $\begin{array}{l}11,8 \\
23,1\end{array}$ & $\begin{array}{l}90 \\
20\end{array}$ & $\begin{array}{l}88,2 \\
76,9\end{array}$ & $\begin{array}{c}1,00 \\
0,44(0,14-1,32)\end{array}$ & 0,13 \\
\hline $\begin{array}{l}\text { Estilo de } \\
\quad \text { Vida } \\
\text { Fantástico }\end{array}$ & Excelente & 11 & 22,9 & 73 & $\begin{array}{l}77,1 \\
91,3\end{array}$ & $\begin{array}{c}1,00 \\
3,10(1,11-8,65)\end{array}$ & 0,02 \\
\hline Colesterol & $\begin{array}{l}\text { Não } \\
\text { Sim }\end{array}$ & $\begin{array}{c}16 \\
2\end{array}$ & $\begin{array}{l}14,3 \\
12,5\end{array}$ & $\begin{array}{l}96 \\
14\end{array}$ & $\begin{array}{l}85,7 \\
87,5\end{array}$ & $\begin{array}{c}1,00 \\
1,16(0,24-5,62)\end{array}$ & 0,84 \\
\hline $\begin{array}{l}\text { Problema } \\
\text { na Coluna }\end{array}$ & $\begin{array}{l}\text { Não } \\
\text { Sim }\end{array}$ & $\begin{array}{c}15 \\
3\end{array}$ & $\begin{array}{c}17,4 \\
7,1\end{array}$ & $\begin{array}{l}71 \\
39\end{array}$ & $\begin{array}{l}82,6 \\
92,9\end{array}$ & $\begin{array}{c}1,00 \\
2,74(0,74-10,07)\end{array}$ & 0,11 \\
\hline Depressão & $\begin{array}{l}\text { Sem sintomas } \\
\text { Com sintomas }\end{array}$ & $\begin{array}{c}6 \\
12\end{array}$ & $\begin{array}{l}14,0 \\
14,1\end{array}$ & $\begin{array}{l}37 \\
73\end{array}$ & $\begin{array}{l}86,0 \\
85,9\end{array}$ & $\begin{array}{c}1,00 \\
0,98(0,34-2,83)\end{array}$ & 0,98 \\
\hline
\end{tabular}

\section{Perfil Antropométrico}

$\begin{array}{llcccccc}\text { IMC } & \text { Eutrófico } & 7 & 19,4 & 29 & 80,6 & 1,00 & 0 \\ & \text { Sobrepeso/Obeso } & 11 & 12,0 & 81 & 88,0 & 1,77(0,62-5,02) & 0,27\end{array}$

*associações significativas ao nível de $\mathrm{p}<0,25$.

As variáveis que apresentaram associação com a prática suficiente de atividade física após a regressão logística foram as mulheres apresentaram $73 \%$ de chances a mais de praticarem atividade física de forma suficiente $(\mathrm{p}=0,73)$ ao serem comparadas aos homens. Ao analisar o estado conjugal não ter companheiro foi considerado fator protetor para a pratica suficiente da atividade física $(\mathrm{p}=0,008)$, ou seja, os solteiros têm $80 \%$ de chances a mais para praticarem atividade física suficiente ao comparado com os casados. Ao analisar a qualidade do sono os colaboradores que não tem alteração no sono apresentaram $70 \%$ de chances a mais de praticarem atividade física. Quanto ao estilo de vida, os colaboradores que apresentaram um estilo de vida excelente têm $30 \%$ de chances a mais de praticarem atividade física suficiente $(\mathrm{p}=0,003)$ (Tabela 2).

Tabela 2: Razão de prevalência ajustada para nível de atividade física de acordo com os fatores sociodemográficos e hábitos comportamentais.

\begin{tabular}{clll}
\hline Variáveis & RP $($ IC95\% $)$ & $\mathbf{p}$ \\
\hline
\end{tabular}

Persp. Online: biol. \& saúde, Campos dos Goytacazes, 37 (11) 50-61, 2021

seer.perspectivasonline.com.br 


\section{Fatores sociodemográficos}

Sexo

\section{Hábitos Comportamentais}

Estado conjugal

Sono alterado

Estilo de Vida Fantástico
Com companheiro

Sem companheiro

Não

Sim

Excelente

Regular
Ajustada*

Feminino

1,00

0,07

$2,88(0,90-9,20)$
Masculino
1,00

$0,14(0,03-0,59)$

1,00

0,00

$9,69(1,89-50,31)$

1,00

$11,40(2,34-55,45)$

\section{DISCUSSÃO}

Esse estudo verificou baixa prevalência da prática de atividade física em colaboradores técnicos, no entanto, fatores sociodemográficos e hábitos comportamentais se mantiveram associados ao desfecho final. Na presente pesquisa, foi constatado que $14,1 \%$ da população praticava atividade física suficiente. Esses resultados vão de encontro a estudos feitos por Sousa et al. (2013), no qual 16,4\% da população referiram praticar atividade física suficiente.

A baixa prevalência da prática de atividade física eleva a predisposição para doenças crônicas não transmissíveis, como as doenças cardíacas, acidentes vasculares cerebrais, diabetes dentre outras, levando muitas das vezes á óbito (MARTINS et al., 2017). Dessa forma, tratar o sedentarismo torna-se fundamental para diminuir o número de mortalidades, e o alto custo nos tratamentos voltados para essas patologias (DUMITH et al., 2019).

Após a análise logística multivariada, a variável sexo ficou associada com a variável desfecho, evidenciando que as mulheres praticavam mais atividades físicas ao serem comparadas aos homens. Esses dados corroboram com estudos feitos por Cardoso et al. (2008), no qual as mulheres também mostraram-se mais ativas em relação aos homens. Isso pode ser associado a grande variedade de atividades, principalmente domésticas realizadas pelas mulheres. Além disso, de acordo com Maciel et al. (2019), essa população compõe um grupo que apresenta elevada insatisfação com a imagem corporal, o que por vez caracteriza a busca pela prática de atividade física como uma tentativa de moldar o corpo de acordo com os padrões impostos pelo meio social.

A variável estado conjugal apresentou efeito protetor, evidenciando que os colaboradores casados praticam menos atividade física. Pontes et al. (2019), evidenciaram em seus achados que a maior parte da população estudada que praticavam atividade física era solteira. Esses dados podem ser justificados pela maior disponibilidade de tempo empregada na

Persp. Online: biol. \& saúde, Campos dos Goytacazes, 37 (11) 50-61, 2021 
prática das atividades, além de ser considerada também como instrumento de socialização com outros indivíduos e também de lazer.

Em relação ao sono manteve-se associado com a prática de atividade física, tornando evidente que os colaboradores que não praticam atividade física apresentam pior qualidade de sono. Ropke et al. (2017), em seus estudos já mencionava a escassez de pesquisas que visem analisar a influência da intensidade, duração e o tipo de exercício na qualidade do sono. No entanto, mesmo diante de raras literaturas voltadas a esse tema, esses autores delinearam o papel fundamental da atividade física como fator favorável quanto a percepção subjetiva e objetiva do sono.

O estilo de vida dos colaboradores manteve associados a prática de atividade física, demonstrando que quem não pratica percebe-se com pior estilo de vida. Esse dado corrobora com a análise feita pela Vigilância de Fatores de Risco e Proteção para Doenças Crônicas por Inquérito Telefônico (VIGITEL) em 2017, que menciona que a prática irregular de atividade física pode causar diminuição das capacidades funcionais, o que por vez acarreta em um pior estilo de vida, e consequentemente, na deterioração do estado de saúde.

É importante considerar algumas limitações apresentada após a realização desse estudo. A primeira está relacionada ao delineamento transversal, visto que mediante o curto espaço de tempo não é possível a análise de inferências casuais. A segunda refere-se ao fato do estudo apenas ter abordado uma instituição privada de ensino superior, o que restringiu o tamanho amostral. Todavia, entende-se que as informações proporcionadas por esse estudo sejam úteis, uma vez que a amostra investigada foi aleatória probabilística e representativa da população estudada, chamando a atenção, principalmente de colaboradores técnicos para a presença de um hábito comportamental com altas taxas de mortalidade no Brasil.

\section{CONCLUSÕES}

Conclui-se que o baixo nível de prática de atividade física está relacionado a fatores como sexo, sono e estilo de vida. Além disso, nesse estudo pode-se constatar que os colaboradores casados apresentam efeito protetor para a prática de atividade física. Isso demonstra a importância de se rastrear esses fatores predisponentes e criar estratégias de controle a fim de reverter essa atual situação, visando sempre a melhora na qualidade de vida dessa população.

\section{REFERÊNCIAS}

ANEZ, C.R.R.; REIS, R.S.; PETROSKI, E.L. Versão Brasileira do Questionário "Estilo de Vida Fantástico": Tradução e Validação para Adultos Jovens. Arquivos Brasileiros de Cardiologia, v. 91, n. 2, p. 102-109, 2008.

ARAÚJO, H.E.S.; OLIVEIRA, G.P.C. A atividade física e a qualidade de vida do policial militar de Goiás. Trabalho de conclusão de curso (Curso de Formação Policial) - Comando da academia da polícia Militar de Goiás- CAPM, 2019.

ASSOCIAÇÃO BRASILEIRA PARA ESTUDO DA OBESIDADE E DA SÍNDROME METABÓLICA (ABESO). Diretrizes brasileiras de obesidade 2016. 4.ed. São Paulo, 2016.

Persp. Online: biol. \& saúde, Campos dos Goytacazes, 37 (11) 50-61, 2021

seer.perspectivasonline.com.br 
BATISTA, D.O.; SILVA, L.M.M.; CALOMENI, M.R. Perfil de atividade cerebral e do desempenho funcional e cognitivo de idosos com diferentes níveis de atividades físicas habituais. Perspectivas Online: Biológicas \& Saúde, v. 08, n.27, supp. 2018

BRASIL. Ministério da Saúde. Departamento de Análise de Situação de Saúde. Vigilância de Fatores de Risco e Proteção para doenças crônicas por inquérito telefônico, VIGITEL. Estimativas sobre frequência e distribuição sócio-demográfica de fatores de risco e proteção para doenças crônicas nas capitais dos 26 estados brasileiros e no Distrito Federal em 2017. Brasília. Distrito Federal. 2017.

CARDOSO, A.S.; LEVANDOSKI, G.; MAZO, G.Z.; PRADO, A.P.M.; CARDOSO, L.S. Comparação do nível de atividade física em relação ao gênero de idosos participantes de grupos de convivência. RBCEH, v.5, n.1. p. 9-18, 2008.

CÔRREA, A.C.; OLIVEIRA, M.S.; COELHO, L.R.P.; REZENDE, L.F.C.; KASHIWABARA, T.G.B. Benefícios da atividade física na saúde e qualidade de vida do trabalhador. Medicina Ambulatorial VI com ênfase em medicina do trabalho. In: KASHIWABARA et al. (org.), Montes Claros. Dejan Gráfica e Editora. 2019.

DUMITH, S.C.; MACIEL, F.V.; BORCHARDT, J.L.; ALAM, V.S.; SILVEIRA, F.C.; PAULITSCH, R.G. Preditores e condições de saúde associados à pratica de atividade física moderada e vigorosa em adultos e idoso no sul do Brasil. Revista Brasileira de Epidemiologia, v.22, e190023, 2019.

MACIEL, M.G.; BRUM, G.P.D.B.; COSTA, L.C.F. Imagem corporal e comportamento alimentar entre mulheres em prática de treinamento resistido. Revista Brasileira de Nutrição Esportiva, v. 13, n. 78, p. 159-166, 2019.

MARTINS, R.C.; SILVA, I.C.M.; HALLA, P.C. Atividade física na população rural de Pelotas, RS: prevalência e fatores associados. Revista de Saúde Pública, v. 52, Supl. 1:9s, 2018.

ORGANIZAÇÃO MUNDIAL DA SAÚDE (OMS). OMS: $80 \%$ dos adolescentes no mundo não praticam atividades físicas suficientes. 2018

PEIXOTO, S.V.; MAMBRINI, J.V.M.; FIRMO, J.O.A.; FILHO, A.I.L.; JUNIOR, P.R.B.S.; ANDRADE, F.B.; LIMA-COSTA, M.F. Prática de atividade física entre adultos mais velhos: resultados do ELSI- Brasil. Revista de Saúde Pública, v.52, n.2, 2018.

PONTES, S.S.; SILVA, A.M.; SANTOS, L.M.S.; SOUSA, B.V.N.; OLIVEIRA, E.F. Práticas de atividade física e esporte no Brasil. Revista Brasileira em Promoção da Saúde, v. 32, 2019.

ROPKE, L.M.; SOUZA, A.G.; BERTOZ, A.P.M.; ADRIAZOLA, M.M.; ORTOLAN, E.V. P.; MARTINS, R.H.; LOPES, W.C.; RODRIGUES, C.D.B.; WEBER, S.A.T. Efeito da atividade física na qualidade do sono e qualidade de vida: revisão sistematizada. Archives of Health Investigation, v.6, n.12, p.561-566, 2017.

SANCHEZ, M.A; BRASIL, J.M.M.; FERREIRA, I.A.M. Benefícios de um programa de atividade física para a melhoria da qualidade de vida de idosos no estado do Rio de Janeiro. Revista Brasileira de Ciência do Envelhecimento Humano, v.11, n. 3, p. 209-218, 2014.

Persp. Online: biol. \& saúde, Campos dos Goytacazes, 37 (11) 50-61, 2021

seer.perspectivasonline.com.br 
SOUSA, C.A.; CÉSAR, C.L.G.; BARROS, M.B.A.; CARANDINA, L.; GOLDBAUM, M.; MARCHIONI, D.M.L.; FISBERG, R.M. Prevalência de atividade física no lazer e fatores associados: estudo de base populacional em São Paulo, Brasil, 2008-2009. Cadernos de Saúde Pública, v.29, n. 2, p. 270-282, 2013.

TALARICO NETO, T.; HAHNS JÚNIOR, H.C.; FERNANDES, L.A.; TEIXEIRA-ARROYO, C.; \& SILVA, T.P.F. Nível de atividade física e uso de drogas lícitas em universitários. Perspectivas Online: Biológicas \& Saúde, v.10, n.34, p.58-67, 2020. 